\title{
Choice of Wavelet from Wavelet Families for DWT- DCT-SVD Image Watermarking
}

\author{
Anjul Singh Akash Tayal
}

\begin{abstract}
To achieve the copyright protection in digital images, watermarking is identified as a major technology to protect digital images from illegal manipulation and geometric distortions. In this paper we discuss a digital image watermarking algorithm based on Discrete Wavelet Transform Discrete Cosine Transform -Singular Value Decomposition (DWT-DCT-SVD). Here in this paper we examined and compared various wavelet families such as Haar, Daubechies, Biorthogonal and Coiflets for the watermarking algorithm. The difference in this method, from other traditional methods is that the watermark is embedded in high frequency band. Traditionally it is assumed that for having good robustness, a watermark should be embedded in low or mid frequency.DWT provides scalability, DCT provides compression and SVD offers minimum or no distortion. Choice of wavelets depends on the choice of wavelet function as DWT can be composed of any function that satisfies requirements of multiresolution analysis. In each of these wavelet families we analyzed effects of wavelets on image quality. The simulation results show good performance with respect to robustness against various image processing operations.
\end{abstract}

\section{Keywords}

Digital Image watermarking, Haar, Daubechies, Biorthogonal, Coiflet, High frequency coefficient, robustness

\section{INTRODUCTION}

Day by day as the size of internet grows, there is rapid increase in exchange and transmission of digital information and it require a technique to provide the ways to authenticate users to prove their ownership on digital content and prevents to tampering and distribution of the data illegally by the unauthorized users. Currently many tools are easily available which can modify or duplicate the data either its image, video ,audio or text .So to achieve security requirement the useful technique is known as digital watermarking.

Digital image watermarking is emerged as a solution for protecting the multimedia data by embedding one digital information into another digital information that can identify where the image came from or who has rights to it without changing the content of original information. [1-24].Watermark should be robust so that it cannot degrade the visual perception of an original image. Visible watermarks can be easily distorted or removed by using simple image processing techniques .Visible watermark are logos or some other information that is embedded into the Image so that it is readily visible. However in invisible watermarking, the information is virtually invisible after it is embedded into the image.

There are two ways to implement digital image watermarking i.e. in spatial domain and in transform domain. In spatial domain watermark is embedded by directly modifying pixel values. While in transform domain the signal or image is transformed into discrete coefficients which are then modified to insert the watermark and Inverse transform of the modified coefficients are taken to get back the original signal or image. Discrete Fourier Transform, Discrete Cosine Transform and Discrete Wavelet Transform DWT are known as transform domain methods. Transformed domain methods are proved more robust against image operation. [17], [18].

Different approaches are used to embed a watermark in a cover image. A matrix decomposition technique known as SVD (Singular value decomposition) is widely used in watermarking $[11],[12,[13]$ while other prefers to do watermarking in transform domain Discrete Cosine Transform (DCT)[14],[15] and Discrete Wavelet Transform[h].In some papers [7],[16]the combination of DWT-DCT-SVD is used for watermarking.

\section{BACKGROUND AND THEORY}

\subsection{Singular Value Decomposition (SVD)}

With several applications in signal processing and statistics Singular Value Decomposition is a factorization of a complex matrix,. SVD is generalisation of the spectral theorem to arbitrary, not necessarily square, matrices. The SVD of an image A with size $m \times m$ is given by $A=U S V^{\mathrm{T}}$, where $U$ and $V$ are orthogonal matrices. The matrix $U$ contains a set of orthonormal output basis vector directions for the matrix A. The elements of $\mathrm{S}$ are only nonzero on the diagonal and are called singular values of $\mathrm{A}$. The matrix $\mathrm{V}$ thus contains a set of orthonormal input vector directions for the matrix A. The matrix $\Sigma$ contains the singular values, which can be thought of as scalar "gain controls" by which each corresponding input is multiplied to give a corresponding output. Singular Value Decomposition formula of an image can be written as:

$$
\mathrm{A}=U S V^{\mathrm{T}}=\sum_{i=1}^{r} \sigma_{i} u_{i} v_{i}^{T}
$$

The first $r$ columns of $U$ are the left singular vectors, whereas the $\mathrm{r}$ columns of $V$ are the right singular vectors of image A. The rank of $A$ is $r, S=\operatorname{diag}\left(\sigma_{1}, \sigma_{2} \ldots, \sigma_{n}\right)$ satisfies $\sigma_{1} \geq \sigma_{2} \geq \cdots \geq \sigma_{n}>0$.

The singular values (diagonal matrix's') represents the luminance or colour intensity of the image and the matrices ' $u$ ' and ' $\mathrm{v}$ ' represents the geometry of the image when SVD is applied to the image. The slight variation in the singular values doesn't change the visual perception of the image, that means it have very good stability, now it has been scientifically proved. The variation of its singular value is not greater than 2-norm of disturbance matrix. Largest singular value of the matrix is equal to the 2-norm. Singular values exhibit some more properties like rotation invariance, translation invariance, transposition invariance, etc. These all properties of SVD are much desirable in image watermarking [5], [20]. 


\subsection{Discrete Wavelet Transform (DWT)}

On the basis of the multi-scale wavelet analysis and timefrequency analysis Wavelets are special functions which, in a form analogous to sines and cosines in Fourier analysis, are used as basal functions for representing signals. The fundamental idea of the DWT for a two dimensional signal is as follows. An image is generally split into four bands: middle-low frequency, middle-high frequency, high frequency and low frequency. The sub-band LL represents the coarse-scale DWT coefficients while the sub-bands LH, HL and HH represent the fine-scale of DWT coefficients the low frequency sub-band is a low pass approximation of the original image and includes most energy of the image. The other sub-bands include edge components of horizontal, vertical and diagonal directions at different scale and resolution respectively. The process of decomposition is continued until the desired number of levels determined by the application is reached. Applying IDWT to LL, HL, LH, and HH, we can get four different frequency images that are low frequency image, middle-low frequency image, middle-high frequency image and high frequency image separately It is very suitable to identify the areas in the host image where a watermark can be embedded effectively due to DWT's excellent spatio-frequency localization properties [4],[18],[22].

\subsection{Discrete Cosine Transform (DCT)}

An image can be represented as a sum of sinusoids of varying magnitudes and frequencies in Discrete Cosine Transform (DCT). It is a technique that can convert a time domain signal into frequency domain signal. The lowest frequency coefficients are represented by left topmost corner of the matrix while the highest frequency coefficients represented by the right bottom most corner [19]. DCT coefficients for the transformed output image, from an input image, $\mathrm{x}$ are computed according to Eq. 1 shown below. In the equation, $\mathrm{x}$, is the input image having $\mathrm{N} \mathrm{x}$ $M$ pixels, $x(m, n)$ is the intensity of the pixel in row $m$ and column $\mathrm{n}$

of the image, and $y(u, v)$ is the DCT coefficient in row $u$ and column $\mathrm{v}$ of the DCT matrix.

$$
\begin{aligned}
& y(u, v)=\sqrt{\frac{2}{M}} \sqrt{\frac{2}{N}} \alpha_{u} \alpha_{v} \sum_{u=0}^{M-1} \sum_{v=0}^{N-1} x(m, n) \\
& \cos \frac{(2 m+1) u \pi}{2 M} \cos \frac{(2 n+1) v \pi}{2 N}
\end{aligned}
$$

For $\mathrm{u}, \mathrm{v}=0,1,2 \ldots \ldots \ldots \ldots . . \mathrm{N}-1$

\section{DWT-DCT-SVD Watermarking}

In given DWT-DCT-SVD based watermarking scheme cover image $A$ is $\mathrm{N}^{*} \mathrm{~N}$ and visual watermark $\mathrm{W}$ is $\mathrm{N} / 2 * \mathrm{~N} / 2$ is used. The four different types of wavelet can be used to embed visual watermarks or the same watermark. So for making a robust DWT-DCT-SVD scheme, we analyse a watermarking scheme, in which we embed the watermark in high frequency band and the behaviour of four different types of wavelet haar, daubiechies2, biorthogonal and coiflets for this image watermarking scheme.

Let A be the cover image. Apply one level DWT to decompose the cover image A into four sub-bands (i.e LL, HL, LH, and $\mathrm{HH})$.Then apply DCT to chosen $(\mathrm{HH})$ band. Let ' $B$ ' denote the matrix obtained after applying DCT. Now apply SVD to 'B' (the discrete cosine transformed coefficients of $\mathrm{HH}$ band). Then use DWT to decompose watermark in four bands).After that Apply SVD to DCT coefficients watermark. Now modify the singular values of $B$ using singular values of $W$. At last apply inverse DCT to modified image B.Using inverse DWT to modified DCT image $\mathrm{B}$, we obtain a watermarked image.

Let $\mathrm{A}^{*}$ be the watermarked image. Apply one level DWT to decompose the watermarked image $A^{*}$ into sub-bands. Then apply DCT to chosen $(\mathrm{HH})$ band. Let ' $B$ ') denote the matrix obtained after applying DCT.Now apply SVD to ' $B$ *' (the discrete cosine transformed coefficients of $\mathrm{HH}$ band. After that extract singular values of watermarking. Now we obtain the modified coefficient, apply inverse DCT to modified image $\mathrm{B}^{*}$.Using inverse DWT to modified DCT image $\mathrm{B}^{*}$ we recovered watermark.

\section{CHOICE OF WAVELET FOR WATERMARKING}

The choice of wavelets is crucial and determines the performance in wavelet-based image watermarking. Choice of wavelet function defines the existence or criteria of the choice of wavelet families. The choice of wavelet functions depends on the content and resolution of image. The performance is depends on the choice of wavelets in wavelet-based image watermarking. To determine optimal wavelet, we compare the normalised coefficients for performance of various wavelets on to cover images set.

The families of wavelet used in our experiments are illustrates fig-1.In this paper, we have considered wide variety of test images and the four wavelets namely: Haar, Daubechies2, Biorthogonal, Coiflets1[9], [23],[24]. (a) Haar Wavelet

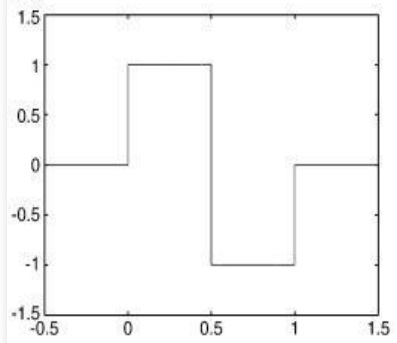

(c) Biothogonal Wavelet

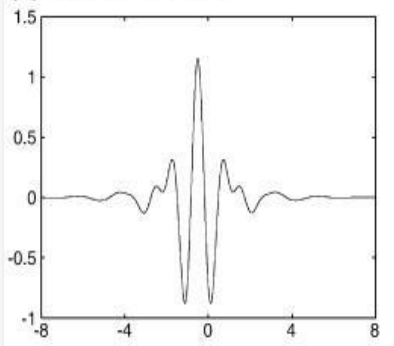

(b) Daubechies Wavelet

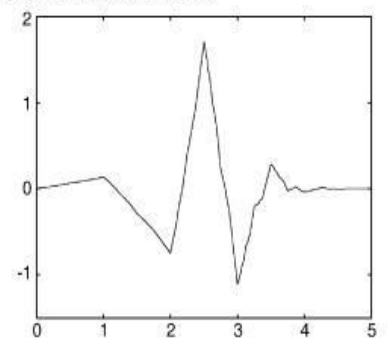

(d) Coiflet Wavelet

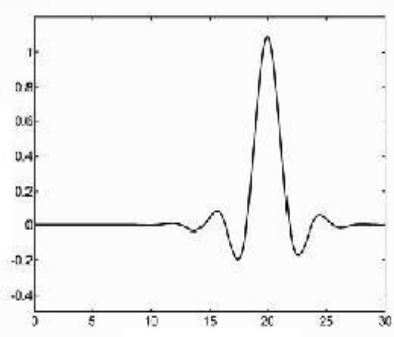

Fig1.(a) Haar (b) Daubechies (c) Biorthogonal (d) Coiflet

Detailed parts of the image can be representing by these waveforms which are shown above. The introduction of these wavelets can be start with the Haar wavelet. It is oldest and simplest wavelet [9]. Haar wavelets are related to a mathematical operation called the Haar transform in discrete forrn. The Haar wavelet operates on data by calculating the sums and differences of adjacent elements. The Haar wavelet operates first on adjacent horizontal elements and then on adjacent vertical elements. One important and nice feature of the Haar 
wavelet transform is that its transform is equal to its inverse. Haar wavelet is discontinuous, and it resembles a step function. In the world of wavelet research, Daubechies is one of the brightest stars invented what are called compactly supported orthonormal wavelets with six coefficients and biorthogonality. All high frequency changes reflects in the high frequency coefficient spectrum, as Daubechies wavelets use overlapping windows. The names of the Daubechies family wavelets are written $\mathrm{dbN}$, where $\mathrm{N}$ is the order, and $\mathrm{db}$ the "surname" of the wavelet. The Daubechies, biorthogonal, and Coiflets are compactly supported orthogonal wavelets [23]. Biorthogonal wavelet exhibits the property of linear phase, which is needed for signal and image reconstruction [1],[2],[3][9],[10]. The interesting properties can be derived by using two wavelets, one for decomposition and the other for reconstruction instead of the same single one [24]. The wavelets are chosen based on their shape and their ability in a particular application. Coiflet wavelet function has $2 \mathrm{~N}$ moments equal to zero and the scaling function has $2 \mathrm{~N}-1$ moments equal to zero [9].

\section{Performance Evaluation}

For qualitative analysis and to evaluate the performance of the algorithm, a similarity measure between the original watermark $w$ and the extracted watermark $w^{\prime}$ is computed by using the normalized correlation (NC) as:

$$
N C=\frac{\sum_{A l l i} w_{i} w_{i}^{\prime}}{\sum_{A l l i} w_{i}^{2}}, w_{i}, w_{i}^{\prime} \in\{-1,1\}
$$

\section{EXPERIMENTAL RESULTS}

We have performed the experiments and analyzed different wavelets. Four wavelets are chooses from the different wavelet families namely: HAAR, DB_2,BIOR_1.1 COIF_1,for the evaluation of the test images To evaluate the performance of the proposed approach, several experiments were conducted on gray-level images "Pepper" of size $512 * 512$ and "Lena" of size $256 * 256$ are used as the cover image and the watermark image shown in Fig.2. and Fig.3. We use scaling factor 0.5 to perform all the experiments.

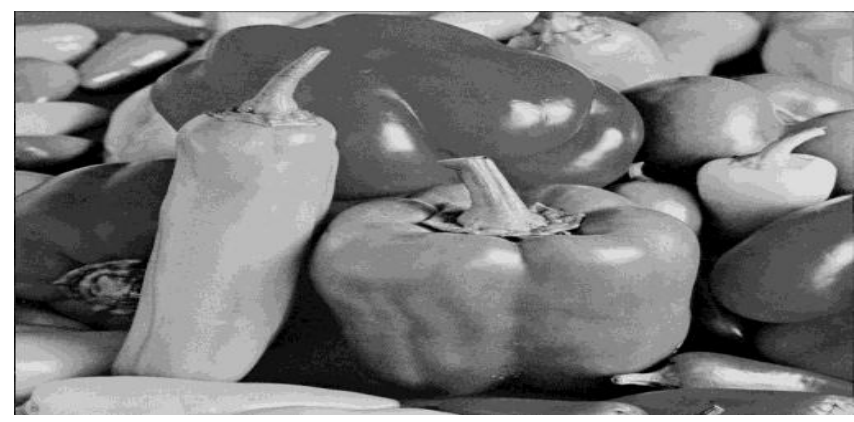

Fig.2. Pepper Image - Cover Image

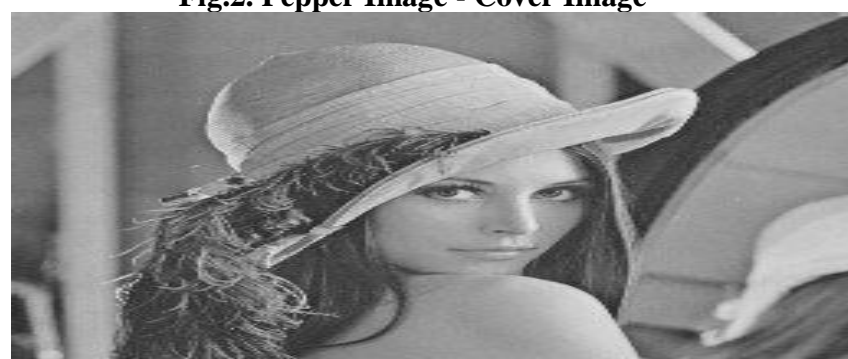

Fig.3.Lena Image - Watermark Image

Proposed scheme is tested under following attacks: Histogram, Poisson noise, Image intensity, Sobel horizontal edge- emphasizing filtering, Speckle noise, Gaussian noise, Rotation, Histogram Equalisation, Salt \& pepper noise, Average filtering, Linear motion, Circular average filtering, Contrast Enhancement, Gaussian low pass filtering for the four wavelets namely: Haar, Daubechies2, Biorthogonal1.1, and Coiflet1.The observed results are shown in Table1 given below.

Table 1: Result - Normalised Correlation for four for the four wavelets namely: Haar, Daubechies2, Biorthogonal1.1, Coiflet1.

\begin{tabular}{|c|c|c|c|c|}
\hline \multirow{2}{*}{ Type of Attack } & \multicolumn{4}{|l|}{$\mathrm{NC}$} \\
\hline & Haar & Bior1.1 & Db2 & Coif1 \\
\hline 1- Histogram & 0.9941 & 0.9951 & 0.9950 & 0.9961 \\
\hline 2- Poisson noise & 0.9754 & 0.9780 & 0.9960 & 0.9769 \\
\hline $\begin{array}{l}\text { 3- Adjust image } \\
\text { intensity }\end{array}$ & 0.9942 & 0.9952 & 0.9951 & 0.9962 \\
\hline $\begin{array}{l}\text { 4- Sobel horizontal } \\
\text { edge-emphasizing } \\
\text { filter }\end{array}$ & 0.9921 & 0.9925 & 0.9944 & 0.996 \\
\hline $\begin{array}{l}\text { 5- Adjust image } \\
\text { intensity }\end{array}$ & 0.9958 & 0.9956 & 0.9963 & 0.9968 \\
\hline $\begin{array}{l}\text { 6- Speckle noise to } \\
\text { image }\end{array}$ & 0.9662 & 0.9687 & 0.9665 & 0.9663 \\
\hline $\begin{array}{l}\text { 7- Gaussian noise to } \\
\text { image }\end{array}$ & 0.8893 & 0.8906 & 0.8889 & 0.8852 \\
\hline $\begin{array}{l}\text { 8- Rotate image } 5 \\
\text { degree }\end{array}$ & 0.9946 & 0.9944 & 0.9949 & 0.9953 \\
\hline $\begin{array}{l}\text { 9- Histogram } \\
\text { Equalisation } \\
\end{array}$ & 0.9823 & 0.9869 & 0.9847 & 0.9876 \\
\hline $\begin{array}{l}\text { 10- Salt \& pepper } \\
\text { noise to image }\end{array}$ & 0.9636 & 0.9659 & 0.9625 & 0.9616 \\
\hline $\begin{array}{l}\text { 11- Average filter[3 } \\
\text { 3] }\end{array}$ & 0.9909 & 0.9909 & 0.9909 & 0.9914 \\
\hline $\begin{array}{l}\text { 12- Average filter[5 } \\
\text { 5] }\end{array}$ & 0.9896 & 0.9896 & 0.99 & 0.9909 \\
\hline $\begin{array}{l}\text { 13- Average filter[7 } \\
\text { 7] }\end{array}$ & 0.9891 & 0.9891 & 0.9899 & 0.9908 \\
\hline 14- Linear motion & 0.9912 & 0.991 & 0.992 & 0.9927 \\
\hline $\begin{array}{l}\text { 15- Circular } \\
\text { averaging filter }\end{array}$ & 0.9887 & 0.9887 & 0.9898 & 0.9908 \\
\hline $\begin{array}{l}\text { 16- Contrast } \\
\text { Enhancement }\end{array}$ & 0.9338 & 0.9511 & 0.9473 & 0.9613 \\
\hline $\begin{array}{l}\text { 17- Gaussian low } \\
\text { pass filter }\end{array}$ & 0.9956 & 0.9956 & 0.9963 & 0.9969 \\
\hline
\end{tabular}

Table.2.(a), (b) and (c) shows the value of normalised correlation of extracted watermark image after the watermarked image attacked by three noise addition for the four wavelets namely: Haar, Daubechies2, Biorthogonal1.1, Coiflets1 .

Fig.4.(a), (b) and (c) shows behaviour of normalised correlation Under the Salt \& pepper noise attack the NC (Normalised correlation) is decreased with the increment in noise density. Under the Gaussian noise attack the NC (Normalised correlation) is decreased with the increment in variance and mean zero. Under the Speckle noise attack the NC (Normalised correlation) is decreased with the increment in variance. 
Table 2(a). Normalized Correlation for Salt \& Pepper Attack

\begin{tabular}{|c|c|c|c|c|}
\hline & \multicolumn{4}{|c|}{ Normalized Correlation } \\
\hline Noise Density & haar & bior1.1 & db2 & coif1 \\
\hline 0.01 & 0.9636 & 0.9659 & 0.9625 & 0.9616 \\
\hline 0.02 & 0.9289 & 0.9293 & 0.9290 & 0.9285 \\
\hline 0.03 & 0.8971 & 0.8984 & 0.8975 & 0.8954 \\
\hline 0.04 & 0.8685 & 0.8973 & 0.8664 & 0.8625 \\
\hline 0.05 & 0.8420 & 0.8438 & 0.8419 & 0.8366 \\
\hline 0.06 & 0.8209 & 0.8207 & 0.8153 & 0.8121 \\
\hline 0.07 & 0.7980 & 0.8189 & 0.8007 & 0.7915 \\
\hline 0.08 & 0.7809 & 0.8001 & 0.7797 & 0.7681 \\
\hline
\end{tabular}

Table 2(b). Normalized Correlation for Gaussian Noise Attack

\begin{tabular}{|c|c|c|c|c|}
\hline & \multicolumn{4}{|c|}{ Normalized Correlation } \\
\hline Variance & haar & bior1.1 & db2 & coif1 \\
\hline 0.01 & 0.8893 & 0.8906 & 0.8889 & 0.8852 \\
\hline 0.02 & 0.8116 & 0.8873 & 0.8886 & 0.8014 \\
\hline 0.03 & 0.7563 & 0.8106 & 0.8890 & 0.7427 \\
\hline 0.04 & 0.7139 & 0.7560 & 0.8892 & 0.6974 \\
\hline 0.05 & 0.6814 & 0.7134 & 0.8887 & 0.6653 \\
\hline 0.06 & 0.6565 & 0.6815 & 0.8897 & 0.6375 \\
\hline 0.07 & 0.6323 & 0.6544 & 0.8888 & 0.6128 \\
\hline 0.08 & 0.6142 & 0.6336 & 0.8884 & 0.5952 \\
\hline
\end{tabular}

Table 2(c). Normalized Correlation for Speckle Noise Attack

\begin{tabular}{|c|c|c|c|c|}
\hline & \multicolumn{4}{|c|}{ Normalized Correlation } \\
\hline Variance & haar & bior1.1 & db2 & coif1 \\
\hline 0.01 & 0.9662 & 0.9687 & 0.9665 & 0.9663 \\
\hline 0.02 & 0.9659 & 0.9351 & 0.9354 & 0.9329 \\
\hline 0.03 & 0.9057 & 0.9062 & 0.9072 & 0.9030 \\
\hline 0.04 & 0.8808 & 0.8799 & 0.8823 & 0.8753 \\
\hline 0.05 & 0.8569 & 0.8571 & 0.8580 & 0.8494 \\
\hline 0.06 & 0.8370 & 0.8362 & 0.8376 & 0.8277 \\
\hline 0.07 & 0.8191 & 0.8188 & 0.8178 & 0.8077 \\
\hline 0.08 & 0.8013 & 0.802 & 0.8013 & 0.7901 \\
\hline
\end{tabular}

Salt \& Pepper Noise Attack

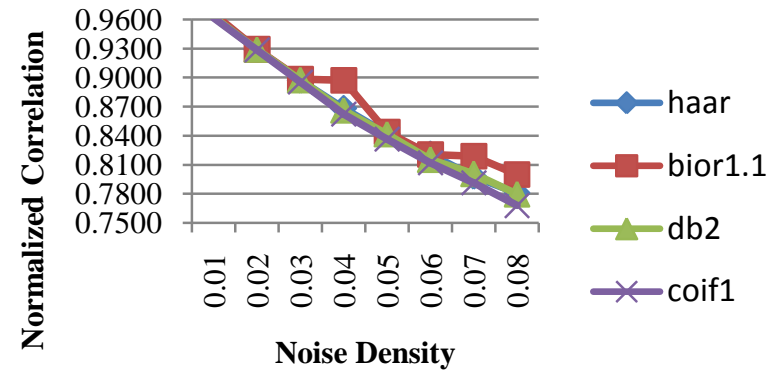

Fig.4 (a) Salt \& Pepper Noise Attack

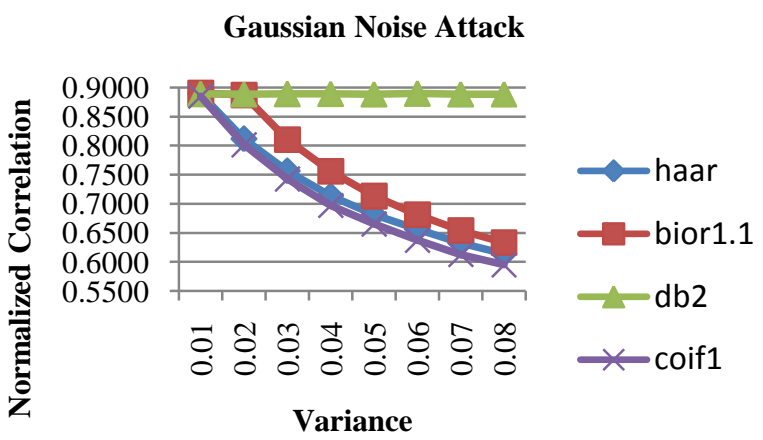

Fig.4 (b) Gaussian Noise Attack

Speckle Noise Attack

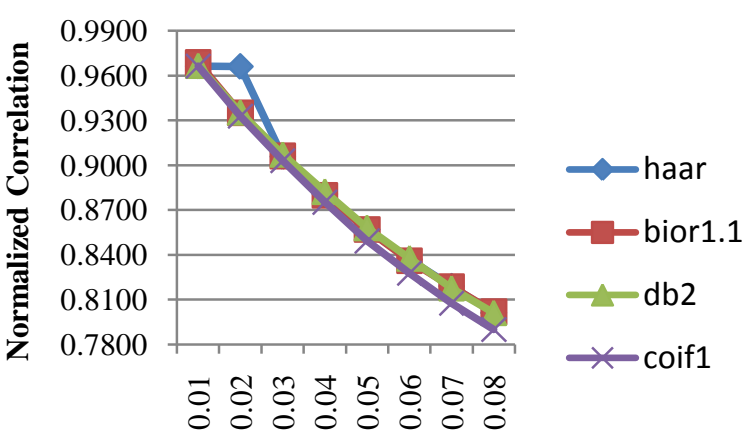

Variance

Fig.4(c) Speckle Noise Attack

Table.3.(a), and (b) shows the value of normalised correlation of extracted watermark image after the watermarked image attacked for the four wavelets namely: Haar, Daubechies2, Biorthogonal1.1, Coiflets1

Fig.5. (a) and (b) Shows robustness of proposed algorithm after Contrast Adjustment attack and Gaussian low pass filtering attack. Normalised correlation of extracted watermark image after the watermarked image attacked by Contrast Adjustment is in between 0.7101 to 0.9203 for Haar, 0.7547 to 0.9338 for Biorthogonal1.1, 0.7151 to 0.9338 for Daubechies2, 0.7236 to 0.9484 for Coiflet1. Under the Gaussian low pass filtering attack Normalised correlation of extracted watermark image is always 
bigger than 0.9914 for the four wavelets namely: Haar, Daubechies2, Biorthogonal1.1, Coiflet1.

Table 3(a). Normalized Correlation for Contrast Adjustment

\begin{tabular}{|c|c|c|c|c|}
\hline & \multicolumn{4}{|c|}{ Normalized Correlation } \\
\hline Alpha & haar & bior1.1 & db2 & coif1 \\
\hline 0.1 & 0.7101 & 0.7547 & 0.7151 & 0.7236 \\
\hline 0.2 & 0.7603 & 0.7944 & 0.7615 & 0.7726 \\
\hline 0.3 & 0.7944 & 0.8286 & 0.8028 & 0.8158 \\
\hline 0.4 & 0.8286 & 0.8581 & 0.8387 & 0.8531 \\
\hline 0.5 & 0.8581 & 0.8828 & 0.8695 & 0.8846 \\
\hline 0.6 & 0.8828 & 0.9034 & 0.8954 & 0.9107 \\
\hline 0.7 & 0.9034 & 0.9203 & 0.9167 & 0.9318 \\
\hline 0.8 & 0.9203 & 0.9338 & 0.9338 & 0.9484 \\
\hline
\end{tabular}

Table 3(b). Normalized Correlation for Low Pass Filter Attack

\begin{tabular}{|c|c|c|c|c|}
\hline & \multicolumn{4}{|c|}{ Normalized Correlation } \\
\hline $\begin{array}{c}\text { Standard } \\
\text { Deviation }\end{array}$ & haar & bior1.1 & db2 & coif1 \\
\hline 0.1 & 0.9936 & 0.9936 & 0.9946 & 0.9958 \\
\hline 0.2 & 0.9936 & 0.9936 & 0.9946 & 0.9958 \\
\hline 0.3 & 0.9937 & 0.9937 & 0.9947 & 0.9958 \\
\hline 0.4 & 0.9956 & 0.9956 & 0.9963 & 0.9969 \\
\hline 0.5 & 0.9952 & 0.9952 & 0.9955 & 0.9957 \\
\hline 0.6 & 0.9935 & 0.9935 & 0.9934 & 0.9935 \\
\hline 0.7 & 0.9923 & 0.9923 & 0.9921 & 0.9921 \\
\hline 0.8 & 0.9917 & 0.9917 & 0.9914 & 0.9915 \\
\hline
\end{tabular}

Fig.5 (a) Contrast Adjustment

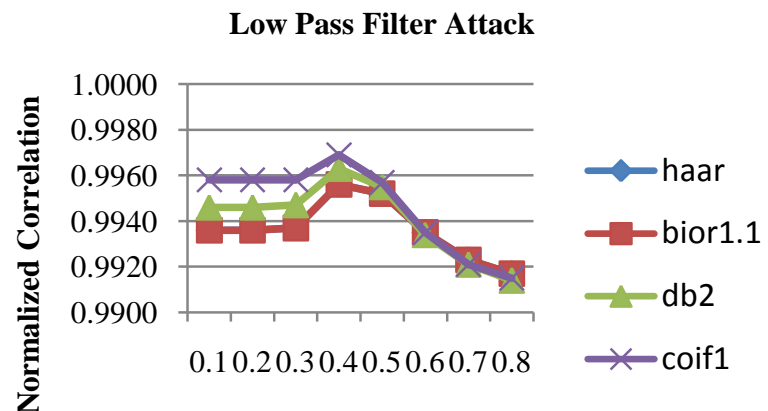

Standard Deviation

Fig.5 (b) Low Pass Filter Attack

\section{CONCLUSION}

By using DWT-DCT-SVD based watermarking scheme a comparative study of different wavelet family has been done using Normalised coefficients, this study give the choice of optimal wavelet for image watermarking. The study shows the robustness of watermark against various image operations and geometric distortions. By analysing the wide range of wavelets namely: Haar, Daubechies2, Biorthogonal1.1, and Coiflet1 we found that Biorthogonal1.1 provide best result for the image quality for salt and pepper noise attack. The wavelet Daubechies2 shows best performance for watermarking under Gaussian noise attack for the cover images. For speckle noise attack haar gives best performance for the given DWT-DCTSVD based Watermarking. The wavelet BIOR_1.1 and Coiflet1 provides competitive performance for the contrast adjustment attack. While for Low pass filter attack Coiflet1 is best choice as shown by the results. Hence, conclusively we can say that the "best wavelet" choice for image watermarking is dependent on the image content and desired image quality. So it is quite correct to say that choice of wavelet depends not only on the size of the image but also on the content of the image for getting a desired image quality and results. With excellent result in high frequency band for the wavelets namely: Haar, Daubechies2, Biorthogonal1.1, and Coiflet1, for further research we would be applying two or more level DWT decomposition so that we can embed small sized watermark and also inquire to embed multiple watermarks so that watermarked image can be more resistant to the image processing attacks.

\section{REFERENCES}

[1] Rajendra S. Shekhawat, Sivavenkateswara Rao V. V. K. Srivastava. A Biorthogonal Wavelet Transform Based Robust Watermarking Scheme[C] IEEE 2012, 978-1-46731515-9/12.

[2] Achintya Singhal, Rudra Pratap Singh, Monal Tenguria Comparision Of Different Wavelets For of Coloured Images watermarking [C]IEEE 2011, 978-1-4244-8679-3.

[3] Li Chunhua,Fu Li,Adaptive Image Watermarking Algorithm Based on Biorthogonal Wavelet Transform [C] IEEE 2011, 978-1-4244-8694-6/11.

[4] Tang Xn, Wen Q, Nian G J. An improved robust watermarking technique in wavelet domain [J]. IEEE, 2010, 978-0-7695-4008-5/10.

[5] Kapre Bhagyashri S, Joshi M.Y. Robust Image Watermarking based on Singular Value Decomposition and Discrete Wavelet Transform[C]. IEEE, 2010, 978-1-4244$5540-9 / 10$

[6] H.T. Wu and Y.M.Cheung, Reversible watermarking by modulation and Security enhancement, IEEE Trans.Instum.Meas., vol.59, no.1, pp221-228, Jan.2010

[7] S S Bedi, Ashwani Kumar, and Piyush Kapoor," Robust Secure SVD Based DCT - DWT Oriented Watermarking Technique for Image Authentication", in International Conference on IT, March 2009, Thailand

[8] L. Zhang, A. Li, "Robust watermarking scheme based on singular value of decomposition in DWT domain," in Proc. Asia-Pacific Conf. Information Processing (APCIP '09), vol. 2, Shenzhen, July 2009, pp. 19-22.

[9] Evelyn Brannock, Michael Weeks, Robert Harrison Watermarking with Wavelets: Simplicity Leads to Robustness [C] IEEE 2008, 978-1-4244-1884-8/08

[10] S. Marusic, D. B. H. Tay, G. Deng and M. Palaniswami, Even Length Biorthoganal wavelets for Digital Watermarking, [C] IEEE 2005, 0-7803-9243-4/05 
[11] X. Zhu, J. Zhao, H. Xu, "A digital watermarking algorithm and implementation based on improved SVD," in Proc. 18th Int. Conf.Pattern Recognition (ICPR '06), vol. 3, Hong Kong, 2006, pp. 651-656.

[12] D. Stanescu, D. Borca, V. Groza, M. Stratulat, "A hybrid watermarking technique using singular value decomposition," in Proc. IEEE Int. Workshop Haptic Audio visual Environments and Games (HAVE '08), Ottawa, Ont., October 2008, pp. 166-170.

[13] X. Sun, J. Liu, J. Sun, Q. Zhang, W. Ji, "A robust image watermarking scheme based on the relationship of SVD," in Proc. Int.Conf. Intelligent Information Hiding and Multimedia Signal Processing (IIHMSP '08), Harbin, August 2008, pp. 731-734.

[14]Z. Rui-mei, L. Hua, P. Hua-wei, H. Bo-ning, "A blind watermarking algorithm based on DCT," in Proc. 2nd Int. Symp. Intelligent Information Technology Application (IITA '08), vol. 3, Shanghai, Dec. 2008, pp. 821-824.

[15] J. Xiao, Y. Wang, "Toward a better understanding of DCT coefficients in watermarking," in Pacific-Asia Workshop onComputational Intelligence and Industrial Application (PACIIA '08), vol. 2, Wuhan, December 2008, pp. 206209.

[16] Ben Wang; Jinkou Ding; Qiaoyan Wen; Xin Liao; Cuixiang Liu,Animage watermarking algorithm based on DWT-DCT and SVD IEEE10.1109/ICNIDC.2009.5360866

[17] K. A. Navas, M. C. Ajay, M. Lekshmi, T. S. Archana, Sasikumar, "DWT-DCT-SVD based watermarking," in Proc. 3rd Int. Conf. Communication Systems Software and Middleware and Workshops (COMSWARE '08), Bangalore, January 2008, pp. 271-274.

[18] E.Ganic and A.M. Eskiciogulu et.al., Robust embedding of Visual Watermarks using DWTSVD Journal of Electronic Imaging, October- December, 2005.

[19] W.C.Chu, DCT based image watermarking using sub sampling. IEEE Trans Multimedia 5, 34-38, 2003.
[20] R.Liu, T.Tan, An SVD-based watermarking scheme for protecting rightful ownership. IEEE Trans. Multimedia, vol.4, no.1, pp, 121-128, 2002.

[21] lngemar I. Cox, Joe Kilian, E Thomson highton. and Talal Shamoon, "Secure spread SpCCINm watermarking for multimedia," IEEE Trans. Image Proc., vol. 6, no. 12. pp. 1673-1687, December 1997.

[22] S. Mallat, "The theory for multiresolution signal decomposition: The wavelet representation," IEEE Trans. Pattern Anal. Mach. Intell., vol. 11, no. 7, pp. 654-693, Jul. 1989.

[23] W. R. Tettler, J. Huffman and D. C. P.Linden, "Application of compactly supported wavelets to image compression", Proceeding SPIE-1244, 1990, pp. 150-160.

[24] E. Yeung, "Image compression using wavelets", Waterloo, Canada N2L3G1, IEEE, CCECE, 1997.

\section{AUTHORS PROFILE}

Anjul Singh received the B.Tech degree from UPTU,Lucknow in 2006, Pursuing M.Tech Degree fromIndira Gandhi Institute of Technology, Delhi.Her research interest include Wavelet Analysis Theory and its application, Image Processing and Digital Image Watermarking.

Akash Tayal received the B.Tech degree from Jamia Millia Islamia, Delhi, \& the M.Tech Degree in Signal Processing from NSIT, Delhi.He is currently an Assistant Professor with Department of Electronics and Communication Engineering, Indira Gandhi Institute of Technology, Delhi. His research interests include Adaptive signal processing, Nanotechnology, Quantum Computing, Wavelet Analysis Theory and its application, Image Processing. 